\title{
Antimicrobial and Antimycobacterial Activity of Cyclostellettamine Alkaloids from Sponge Pachychalina sp.
}

\author{
Jaine H. H. L. de Oliveira ${ }^{1}$, Mirna H. R. Seleghim ${ }^{1}$, Christoph Timm ${ }^{2}$, Achim Grube ${ }^{2}$, \\ Matthias Köck $^{2}$, Gislene G.F. Nascimento ${ }^{3}$, Ana Claudia T. Martins ${ }^{3}$, Elissa G. O. Silva ${ }^{3}$, Ana \\ Olívia de Souza ${ }^{4}$, Paulo R. R. Minarini ${ }^{4}$, Fabio C. S. Galetti ${ }^{5}$, Célio L. Silva ${ }^{5}$, Eduardo Hajdu ${ }^{6}$ \\ and Roberto G. S. Berlinck ${ }^{1, *}$
}

${ }^{1}$ Instituto de Química de São Carlos, Universidade de São Paulo, CP 780, CEP 13560-970, São Carlos, SP, Brazil. (E-mail: jainehh@iris.ufscar.br for Jaine H. H. L. de Oliveira; E-mail: mirna@iqsc.usp.br for Mirna H. R. Seleghim)

${ }^{2}$ Alfred-Wegener-Institut für Polar- und Meeresforschung in der Helmholtz-Gemeinschaft, Am Handelshafen 12,D-27570 Bremerhaven, Germany. (E-mail: agrube@awi-bremerhaven.de for Achim Grube;E-mail: mkoeck@awi-bremerhaven.de for Matthias Köck)

${ }^{3}$ Faculdade de Ciências da Saúde, Universidade Metodista de Piracicaba, Piracaba, SP, Brazil. (E-mail: ggfranco@unimep.br for Gislene G.F. Nascimento)

${ }^{4}$ Laboratório de Bioquímica e Biofísica, Instituto Butantan, São Paulo, Brazil. (E.mail: olívia@butantan.gov.br for Ana Olívia de Souza)

${ }^{5}$ Departamento de Bioquímica e Imunologia, Faculdade de Medicina de Ribeirão Preto, Universidade de São Paulo, Ribeirão Preto, SP, Brazil. (E-mail: galetti@cpt.fmrp.usp.br for Fabio C. S. Galetti)

${ }^{6}$ Museu Nacional, Universidade Federal do Rio de Janeiro, Quinta da Boa Vista, s/n, 20940-040, Rio de Janeiro, RJ, Brazil. (E-mail: hajdu@acd.ufrj.br for Eduardo Hajdu)

* Author to whom correspondence should be addressed; E-mail: rgsberlinck@iqsc.usp.br

Received: 7 December 2005 / Accepted: 18 January 2006 / Published: 20 January 2005

\begin{abstract}
Cyclostellettamines A - F (1 - 6) isolated from the sponge Pachychalina sp. and cyclostellettamines G - I, K and L (7 - 11) obtained by synthesis were evaluated in bioassays of antimicrobial activity against susceptible and antibiotic-resistant Staphylococcus aureus, Pseudomonas aeruginosa and antibiotic-susceptible Escherichia coli and Candida albicans, as well as in antimycobacterial activity against Mycobacterium tuberculosis H37Rv bioassays. The results obtained indicated that cyclostellettamines display different antimicrobial activity depending on the alkyl-chain size, suggesting that, if a mechanism-of action is implied, it is dependent on the distance between the two pyridinium moieties of cyclostellettamines.
\end{abstract}


Keywords: alkaloids, antimycobacterial, antibacterial, Pachychalina sp., sponge

\section{Introduction}

Microbial promoted infectious diseases are a major public health issue worldwide. Among other, these include infection by antibiotic-resistant microorganisms, which are considered very problematic since only few antibiotics currently available are effective against resistant microbial strains [1-3]. One of the most promising classes of antibiotics is inhibitors of bacteria cell wall synthesis, which are largely based on different classes of natural products [4]. Therefore, there is great interest in finding new classes of natural products that may be effective against antibiotic-resistant bacteria. Also, tuberculosis promoted by Mycobacterium tuberculosis is a major cause of mortality. It is estimated that about 2 billion people are currently infected with $M$. tuberculosis. The number of infections by $M$. tuberculosis appear to be increasing, due to co-infection with HIV and the emergence of multiple drug resistant Mycobacterium strains [5,6]. Although several drugs for the treatment of tuberculosis are available, it is a long-term therapy (6 months), which very frequently present side effects. Therefore, there is an urgent need for new anti-tuberculosis drugs that must be effective, less toxic and promote a short period of treatment $[7,8]$.

In addition to microbial promoted infectious diseases, systemic mycoses are also difficult to medicate. In particular, infections by Candida albicans are increasing in the group of immunologically suppressed patients, for which only a few antifungal agents are effective. Therefore, the search for new antifungal compounds active against Candida spp. is of special importance [9].

Marine macro- and microorganisms have proven to be an outstanding source of structurally unique biologically active natural products [10-12]. Marine sponges constitute the main biological group source of bioactive secondary metabolites from the marine environment. In particular, marine sponges of the order Haplosclerida have been known for a long time as a typical source of alkylpyridine and alkylpiperidine alkaloids [13-16]. During our current search for new bioactive compounds from marine sponges, we have recently started to investigate the chemistry of the antimycobacterial, antimicrobial and cytotoxic $\mathrm{MeOH}$ crude extract of the sponge Pachychalina sp. and isolated a new member of the ingenamine family of alkaloids as well as six known and five new cyclostellettamine alkaloids identified by LC-MS/MS analysis [17]. Herein, we report the antimycobacterial and antimicrobial activity against antibiotic-resistant bacteria of pure cyclostellettamines A - F (1 - 6) isolated from Pachychalina sp. as well as of isolated cyclostellettamines G - I, K and L (7 - 11) obtained by total synthesis.

\section{Results and Discussion}

Cyclostellettamines A - F (1 - 6) were isolated as pure entities from the n-BuOH crude extract of Pachychalina sp. obtained previously [17] by chromatography on Sephadex LH20 (MeOH) followed by reversed phase $C_{18}$ HPLC purification, while cyclostellettamines $G-I, K$ and L (7 - 11) were obtained by total synthesis [18]. All compounds were unambiguously identified by HPLC-MS/MS analysis, as previously reported [17]. 


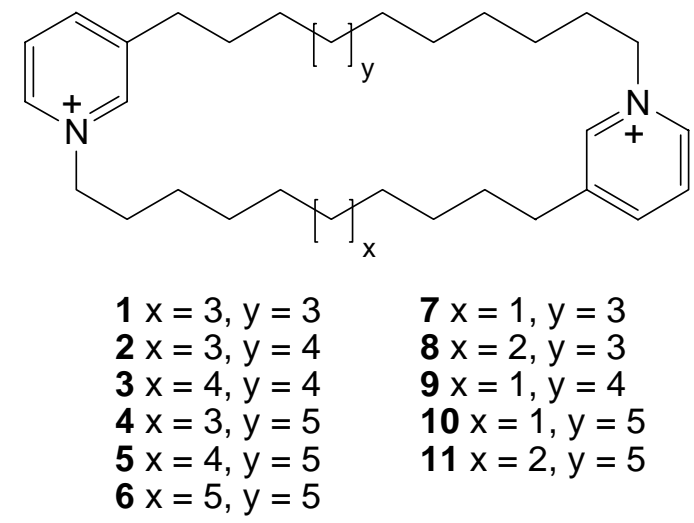

Scheme 1. Structures of cyclostellettamines A - F (1 - 6), G - I, K and L (7 - 11).

Since only a small amount of pure cyclostellettamines was available, they were subjected to antibacterial, antifungal and antimycobacterial bioassays only. The results indicated (Table 1) that cyclostellettamine A (1) was the less active alkaloid in all antimicrobial assays. All other cyclostellettamines were strongly active against antibiotic-susceptible S. aureus ATCC 25923 (strain Sa in table 1) and E. coli ATCC 25922 (Ec), with exception of compound 7 against E. coli. Cyclostellettamine C (3) was the most active against P. aeruginosa ATCC 27853 (strain Pa), cyclostellettamines C (3) and F (6) were the most active against one oxacillin-resistant strain of $S$. aureus (strain ORSa8), and cylostellettamine C (3) against another ORSA strain (strain ORSa108). Cyclostellettamine $\mathrm{F}$ was the most active against both sensitive and antibiotic-resistant $P$. aeruginosa strains (Pa and Pa13). Only cyclostellettamines C - F (3-6) were active against Candida albicans ATCC 10231 (Ca). The results of the antifungal assay suggested that the length of the alkyl chains of cyclostellettamines is an important criterion for activity against Candida. It seems likely that lipophilic alkyl chains can interact with the microbial membrane, while the hydrophilic pyridine moieties may either interact with negatively charged membrane sites. This process may initialize a disorder of the membrane lipids and perhaps an uncontrolled flux of electrolytes, where the positively charged pyridinium moieties may play a crucial role. It has been indeed observed that positively charged lipophillic peptides and cationic vesicles exert a strong antifungal activity against Candida albicans [19-22] through the interaction with the membrane positively charged sites. Cyclostellettamines B (2), C (3), G (7) and L (11) were strongly active against M. tuberculosis H37Rv (MtH37Rv). Overall, cyclostellettamine C (3) was the most active alkaloid. It is interesting to observe that, with exception to S. aureus ATCC 25923 (Sa) and E. coli ATCC 25922 (Ec), all other microorganisms were sensitive to the cyclostellettamines antimicrobial activity depending on the size of the alkyl chains bridging the two alkylpyridinium groups, a result that suggests a mechanism-based activity related to the size of the alkyl chains.

In particular, the results of antimycobacterial activity against $M$. tuberculosis $\mathrm{H} 37 \mathrm{Rv}$ were suggestive that cyclostellettamines with larger alkyl chains, such as compounds $\mathbf{4}, \mathbf{5}$ and $\mathbf{6}$, were less active than compounds with smaller alkyl chains as linkers of the two pyridinium moieties, although cyclostellettamine A (1) is an exception. A similar alkyl chain size-dependent activity was observed for cyclostellettamines A - F (1-6) as inhibitors of the binding of methyl quinuclidinyl benzilate to muscarine acetylcholine receptors when these compounds have been originally reported from the sponge Stelletta maxima [23]. In this case, cyclostellettamine A (1) was the most active and 
cyclostellettamine F (6) the less active alkaloid. Apparently, a correct positioning of the charged pyridinium moieties is necessary to maximize the activity in different bioassays. The results observed in our bioassays suggest a mechanism-based antimicrobial action for the cyclostellettamine dimeric alkylpyridinium alkaloids, differently than observed for the structurally-related oligomeric and polymeric halitoxins, which display potent biological activities in several different bioassays irrespective of their average size, and are considered as nuisance compounds [24]. However, since we have not attempted to simulate the distance between the two pyridinium rings in each cyclostellettamine, our mechanism of action proposal is suggestive only. It should be also noted that since Gram-positive (S. aureus) and Gram-negative (E. coli, P. aeruginosa) bacteria have different membrane properties, cyclostellettamines might certainly present distinct modes of interactions with the respective bacterial membranes. Further structure-activity studies on cyclostellettamine alkaloids may clarify if there is or not a mechanism-based relationship of their biological activities.

Table 1. Minimal inhibitory concentration (MIC, in $\mu \mathrm{g} / \mathrm{mL}$ ) of the antimicrobial activity of cyclostellettamine alkaloids on sensitive and antibiotic-resistant microbial strains*.

\begin{tabular}{ccccccccccc}
\hline \multirow{2}{*}{ Alkaloids } & alkyl chains & \multicolumn{10}{c}{ Microbial strains } \\
\cline { 3 - 10 } & $\boldsymbol{\Sigma}\left(\mathbf{C H}_{\mathbf{2}} \mathbf{n}_{\mathbf{n}}\right.$ & $\mathbf{S a}$ & $\mathbf{E c}$ & $\mathbf{P a}$ & $\mathbf{O R S a 8}$ & $\mathbf{O R S a 1 0 8}$ & Pa13 & PaP1 & $\mathbf{C a}$ & $\mathbf{M t H 3 7 R v}$ \\
\hline $\mathbf{1}$ & $24(12+12)$ & 56.3 & 56.3 & 112.5 & 56.3 & 225 & 112.5 & 450 & $*$ & 32 \\
$\mathbf{2}$ & $25(12+13)$ & 0.5 & 2 & 31.3 & 62.5 & 7.8 & 31.3 & 62.5 & $*$ & 4.0 \\
$\mathbf{3}$ & $26(13+13)$ & 1.2 & 1.2 & 8.6 & 1.8 & 4.7 & 18.8 & 18.8 & 0.12 & 4.0 \\
$\mathbf{4}$ & $26(12+14)$ & 0.3 & 2.1 & 16.4 & 16.4 & 8.2 & 16.4 & 16.4 & 0.7 & 8.0 \\
$\mathbf{5}$ & $27(13+14)$ & 2.3 & 2.4 & 18.8 & 9.4 & 9.4 & 9.4 & 18.8 & 0.7 & 11.0 \\
$\mathbf{6}$ & $28(14+14)$ & 0.6 & 9.4 & 75 & 0.24 & 75 & 9.4 & 4.7 & 0.7 & 8.0 \\
$\mathbf{7}$ & $22(10+12)$ & 0.6 & 39.1 & 156.3 & 39.1 & 78.1 & 156.3 & 156.3 & + & 4.6 \\
$\mathbf{8}$ & $23(11+12)$ & 2.4 & 9.5 & 312.5 & 78.1 & 625 & 312.5 & 312.5 & + & 9.3 \\
$\mathbf{9}$ & $23(10+13)$ & 1.1 & 8.8 & 40.6 & 35.2 & 35.2 & 140.6 & 17.6 & + & 6.6 \\
$\mathbf{1 0}$ & $24(10+14)$ & 1.7 & 1.7 & 27.3 & 437.5 & 875 & 13.7 & 27.3 & + & 5.3 \\
$\mathbf{1 1}$ & $25(11+14)$ & 0.8 & 3.2 & 51.6 & 51.6 & 25.8 & 51.6 & 51.6 & + & 4.6 \\
\hline
\end{tabular}

* Microbial strains: Sa.S. aureus (ATCC 25923) - Gram positive; Ec. E. Coli (ATCC 25922) - Gram negative; Pa. Pseudomonas aeruginosa (ATCC 27853) - Gram negative; ORSa8. Oxacillin-resistant S. aureus strain 8 - Gram positive; ORSa108. Oxacillin-resistant S. aureus strain 108 Gram positive; Pa13. P. aeruginosa 13 (biofilm producer and resistant to several antibiotics); PaP1.P. aeruginosa P1 (biofilm producer and resistant to several antibiotics); Ca. Candida. Albicans (ATCC 10 231) yeast; MtH37Rv. Mycobacterium tuberculosis H37Rv. +: microbial growth with no antibiotic activity detected; * not tested due to insufficient amount of material.

In conclusion, the isolation of the known cyclostellettamines A - F $(\mathbf{1}-\mathbf{6})$ from the sponge Pachychalina sp. and the synthesis of the new cyclostellettamines G - I, K and L (7 - 11) provided enough material for evaluation of antibacterial, antifungal and antimycobacterial activity of these alkaloids. The results obtained indicated that the size of the alkyl chain linker between the two pyridinium aromatic rings seems to be related to the activity level of the cyclostllettamine alkaloids, 
suggesting a mechanism-of-action based on the distance of the charged moieties, possibly through interaction with ionic membrane sites. Further pharmacological and biophysical studies are necessary to clarify such hypotheses.

\section{Experimental}

\section{General}

General experimental procedures for isolation and identification of cyclostellettamines have been previously reported [17].

\section{Animal material}

The sponge Pachychalina sp. was collected in Ilha do Pai (Father’s Island), Niterói, Rio de Janeiro state ( $\left.22^{\circ} 59.205^{\prime} \mathrm{S}-43^{\circ} 05.252^{\prime} \mathrm{W}\right)$, on May 9th 2000 at 10-15 m depth, and immediately immersed in EtOH. The whole material was shipped to the Instituto de Química de São Carlos, Universidade de São Paulo. Voucher specimens are deposited at the Museu Nacional (MNRJ 3098 e 3099).

\section{Extraction and isolation}

The sponge Pachychalina sp. was processed as previoulsy described [17]. A further amount of the $\mathrm{n}$ - $\mathrm{BuOH}$ extract was separated exactly as desbribed in our original procedure, to give additional amounts of the the alkylpyridinium alkaloids fractions AmNL1FN5a and AmNL4a [17]. These fractions were purified by HPLC (Inertsil ODS-2, $125 \AA$, $5 \mu \mathrm{m}, 250$ x $9.4 \mathrm{~mm}$; 43:57 $\mathrm{MeCN}^{-\mathrm{H}_{2} \mathrm{O}+}$ $0.1 \%$ trifluoroacetic acid; flow rate: $1 \mathrm{~mL} / \mathrm{min} ; \lambda_{\max } 266 \mathrm{~nm}$ ) to give $2.5 \mathrm{mg}$ of $\mathbf{1}, 5.4 \mathrm{mg}$ of $2,3.4 \mathrm{mg}$ of 3, $3.6 \mathrm{mg}$ of $\mathbf{4}, 4.1 \mathrm{mg}$ of $\mathbf{5}$ and $3.0 \mathrm{mg}$ of $\mathbf{6}$. Pure compounds were identified by our previously reported HPLC-MS/MS analysis method [17]. Synthetic cyclostellettamines G - I, K and L were prepared as recently described [18].

Antibacterial and antifungal assays

Procedures for the antimicrobial assay against $S$. aureus ATCC 25923, E. coli ATCC 25922, $P$. aeruginosa ATCC 27853, oxacillin-resistant S. aureus strain 8, oxacillin-resistant S. aureus strain 108, P. aeruginosa strain 13, P. aeruginosa strain P1 and C. albicans ATCC 10231 were performed using the reference broth microdilution assay, adapted from the National Committee for Clinical Laboratory Standards (USA) and from Pettit et al. [25], as it follows. Isolated colonies from overnight cultures were suspended and diluted as recommended to yield final inocula of approximately $10^{5} \mathrm{CFU} / \mathrm{mL}$. Tests were performed in sterile 96-well microplates by dispensing into each well a total volume of 100 $\mu \mathrm{L}$ (40 $\mu \mathrm{L}$ de medium $+20 \mu \mathrm{L}$ de inocula $+40 \mu \mathrm{L}$ substance solution dilutes in water) and incubated $24 \mathrm{~h}$ at $37{ }^{\circ} \mathrm{C}$. Microorganism growths were determined by absorbance measurement at $620 \mathrm{~nm}$ in an automated microplate reader (Labsystem, Multikan MS model). The MIC was defined as the lowest concentration of drug that inhibited growth. As control it was used microbial cultures growth without the addition of the tested substance and all samples were assayed in replicate. 
Antimycobacterial assay against Mycobacterium tuberculosis $H 37 R v$

The antimycobacterial activity of crude extracts was assayed against $M$. tuberculosis H37Rv ATCC 27294 by the microplate Alamar Blue assay [22]. Mycobacteria were grown on Loweinstein-Jensen medium at $37^{\circ} \mathrm{C}$ and the concentration adjusted to a bacterial density corresponding to $1.0 \mathrm{McFarland}$ turbidity standard $\left(1 \times 10^{7} \mathrm{cell} / \mathrm{mL}\right)$, that was further diluted 1:25 in Middlebrook 7H9 broth medium before the inoculation $\left(4 \times 10^{5}\right.$ mycobacteria/mL). Stock solutions of the crude extracts were diluted in DMSO at concentrations ranging from 1.0 to $80 \mathrm{mg} / \mathrm{mL}$, sterilized by passage through a $0.22 \mu \mathrm{m}$ PFTE filter (Millex-FG, Millipore) and diluted 1:10 in Middlebrook 7H9 broth. The diluted samples were aliquoted and all the samples were stored at $-20^{\circ} \mathrm{C}$ until necessary. Serial dilutions (of the 1:10 solution) were performed in Middlebrook 7H9 broth medium, in a microplate of 96 wells, to obtain $100 \mu \mathrm{L}$ of solution in each well. The higher concentration of DMSO was 2.5\%. M. tuberculosis $\mathrm{H} 37 \mathrm{Rv}$ was added to each well containing the samples and the microplate incubated at $37^{\circ} \mathrm{C}$ in a humidified chamber. Control wells consisting of either M. tuberculosis only (MB) or medium only (M), as well as those containing crude extract samples $(100 \mu \mathrm{L})$ ranging from 0.01 to $2.0 \mathrm{mg} / \mathrm{mL}$, were inoculated with $100 \mu \mathrm{L}$ of a diluted suspension of $M$. tuberculosis $\left(4 \times 10^{5} \mathrm{cell} / \mathrm{mL}\right)$. The microplate was incubated at $37^{\circ} \mathrm{C}$ for 6 days. Afterwards, $25 \mu \mathrm{L}$ of a mixture $1: 1(\mathrm{v} / \mathrm{v})$ of $10 \mathrm{x}$ Alamar Blue reagent and $10 \%$ Tween 80 were added to the wells and the plates were reincubated at $37^{\circ} \mathrm{C}$. After 24 hours, a change in color from blue to pink was observed in the wells where the mycobacteria grew. The visual minimal inhibitory concentration (MIC) was defined as the lowest drug concentration that prevented a color change from blue to pink. Rifampicin was used as a reference. Assays were performed in triplicate.

\section{Acknowledgements}

Financial support was provided to R.G.S.B by grants from FAPESP (01/03095-5) and from NIH (CA 67786), to whom the authors are gratefully indebted. J.H.H.L.O. also thanks FAPESP for a scholarship.

\section{References and Notes}

1. Andersson, D. I. Persistence of antibiotic resistant bacteria. Curr. Opin. Microbiol. 2003, 6, 452456.

2. DaSilva, E.; M. Iaccarino, M. Emerging diseases: a global threat. Biotech. Adv. 1999, 17, 363-384.

3. Projan, S. J. Why is big Pharma getting out of antibacterial drug discovery? Curr. Opin. Microbiol. 2003, 6, 427-430.

4. Silver, L. L. Novel inhibitors of bacterial cell wall synthesis. Curr. Opin. Microbiol. 2003, 6, 431438.

5. Tripathi R. P.; Tewari, N.; Dwivedi, N.; Tiwari, V. K. Fighting tuberculosis: an old disease with new challenges. Med. Res. Rev. 2005, 25, 93-131.

6. Lawn S. D., Bekker, L.G.; Miller, R. F. Immune reconstitution disease associated with mycobacterial infections in HIV-infected individuals receiving antiretrovirals. Lancet Inf. Dis. 2005, 5, 361-373. 
7. Ballell, L.; Field, R. A.; Duncan, K.; Young, R. J. New small-molecule synthetic antimycobacterials. Antimic. Agent. Chemoth. 2005, 49, 2153-2163.

8. Zhang, Y. The magic bullets and tuberculosis drug targets. Ann. Rev. Pharm. Tox. 2005, 45, 52964.

9. Kossuga, M. H.; MacMillan, J. B.; Rogers, E. W.; Molinski, T. F.; Nascimento, G. G. F.; Rocha, R. M.; Berlinck, R. G. S. (2S,3R)-2-Aminododecan-3-ol, a new antifungal agent from the ascidian Clavelina oblonga, J. Nat. Prod. 2004, 67, 1879-1881, and references therein.

10. Blunt, J. W.; Copp, B. R.; Munro, M. H. G.; Northcote, P. T.; Prinsep, M. R. Marine natural products. Nat. Prod. Rep. 2005, 22, 15-61.

11. Mayer, A. M. S.; Gustafson, K. R. Marine pharmacology in 2001-2: antitumour and cytotoxic compounds. Eur. J. Cancer. 2004, 40, 2676-2704.

12. Mayer, A. M. S.; Hamann, M. T. Marine pharmacology in 1999: compounds with antibacterial, anticoagulant, antifungal, anthelmintic, anti-inflammatory, antiplatelet, antiprotozoal. and antiviral activities affecting the cardiovascular, endocrine, immune and nervous systems, and other miscellaneous mechanisms of action. Comp. Biochem. Physiol. C - Toxicol. Pharm. 2002, 132, 315-339.

13. Andersen, R. J.; van Soest, R. W. M.; Kong, F. 3-Alkylpiperidine alkaloids isolated from marine sponges in the order Haplosclerida, In Alkaloids: Chemical and Biological Perspectives; Pelletier, S. W., Ed.; Pergamon Press: New York, 1996; Vol. 10, pp 301-355.

14. Almeida, A. M. P.; Berlinck, R. G. S.; Hajdu, E. Alcalóides alquilpiridínicos de esponjas marinhas. Química Nova 1997, 20, 170-185.

15. Sepcic, K. J. Bioactive alkylpyridinium compounds from marine sponges. J. Toxicol. - Toxin. Rev. 2000, 19, 139-160.

16. Rodriguez, J. Polycyclic amine alkaloids (3-alkylpiperidine alkaloids), novel marine bioactive compounds: structure, synthesis and biochemical aspects. Stud. Nat. Prod. Chem. 2000, 24, 573581.

17. Oliveira, J. H. H. L., Grube, A., Köck, M., Berlinck, R. G. S., Macedo, M. L., Ferreira, A. G., Hajdu, E. Ingenamine G and cyclostellettamines G-I, K, and L from the new Brazilian species of marine sponge Pachychalina sp. J. Nat. Prod., 2004, 67, 1685-1689.

18. Grube, A.; Timm, C.; Köck, M. Synthesis and mass spectrometric analysis of cyclostellettamines H, I, K and L. Eur. J. Org. Chem. 2006, in press (doi: 10.1002/ejoc.200500208).

19. van der Kraan, M. I. A.; Nazmi, K.; Teeken, A.; Groenink, J.; van 't Hof, W.; Veerman, E. C. I.; Bolscher, J. G. M.; Amerongen, A. V. N. Lactoferrampin, an antimicrobial peptide of bovine lactoferrin, exerts its candidacidal activity by a cluster of positively charged residues at the Cterminus in combination with a helix-facilitating N-terminal part. Biol. Chem. 2005, 386, 137-142.

20. Situ, H.; Wei, G. X.; Smith, C. J.; Mashhoon, S.; Bobek, L. A. Human salivary MUC7 mucin peptides: effect of size, charge and cysteine residues on antifungal activity. Biochem. J. 2003, 375, 175-182.

21. Hong, S. Y.; Park, T. G.; Lee, K. H. The effect of charge increase on the specificity and activity of a short antimicrobial peptide, Peptides 2001, 22, 1669-1674.

22. Carnpanha, M. T. N.; Mamizuka, E. M.; Carmona-Ribeiro, A. M. Interactions between cationic vesicles and Candida albicans, J. Phys. Chem. 2001, 105, 8230-8236. 
23. Fusetani, N., Asai, N., Matsunaga, S., Honda, K. and Yasumuro, K. Cyclostellettamines A - F, pyridine alkaloids which inhibit binding of methyl quinuclidyl benzilate (QNB) to muscarinic acetylcholine receptors, from the marine sponge, Stelletta maxima. Tetrahedron Lett. 1994, 35, 3967-3970.

24. Berlinck, R. G. S., Hajdu, E., da Rocha, R.M., de Oliveira, J.H.H.L., Hernandez, I.L.C., Seleghim, M.H.R., Granato, A.C., de Almeida, E.V.R., Nunez, C.V., Muricy, G., Peixinho, S., Pessoa, C., Moraes, M.O., Cavalcanti, B.C., Nascimento, G.G.F., Thiemann, O., Silva, M., Souza, A.O., Silva, C.L., Minarini, P.R.R. Challenges and rewards of research in marine natural products chemistry in Brazil, J. Nat. Prod. 2004, 67, 510-522.

25. Pettit, R. K., Fakoury, R. B., Knight, J.C., Weber, C. A., Pettit, G. R., Cage, G.D., Pon, S. Antibacterial activity of the marine sponge constituent cribrostatin 6. J. Med Microbiol. 2004, 53, 61-65.

26. Collins, L.A., Franzblau, S.G. Microplate Alamar Blue assay versus BACTEC 460 system for high-throughput screening of compounds against Mycobacterium tuberculosis and Mycobacterium avium. Antimicrob. Agents Chemother. 1997, 41, 1004-1009.

Samples availability: not available.

(C) 2006 by MDPI (http://www.mdpi.org). Reproduction is permitted for noncommercial purposes. 